\title{
Quasi-ternary nanoparticle superlattices through nanoparticle design $* *$
}

\author{
By Elena V. Shevchenko*, Jeffrey B. Kortright, Dmitri V. Talapin, Shaul Aloni and A. Paul Alivisatos*
}

Individual nanoscale building blocks exhibit a wide range of size-dependent properties, since their size can be tuned over known characteristic length scales of bulk materials. In the last several years, the possibility of combining different materials in the form of two and three component nanoparticles (NPs) has been extensively explored. ${ }^{[1-10]}$ Also multi-component materials can be obtained via self-assembly of NPs from their binary colloidal mixtures. ${ }^{[11-15]}$ These new nanocrystal solids may possess tunable collective properties that originate from interactions between size and composition controlled building blocks. Exchange coupling between neighboring NPs of magnetically soft and hard materials enhances the magnetic energy product of the nanocomposite material. ${ }^{[16,17]}$ Randomly mixed solids of small and large semiconducting CdSe NPs revealed enhancement of photoluminescence intensity of large semiconductor particles accompanied by quenching of photoluminescence of the small particles because of long-range resonant transfer of electronic excitations from the more electronically confined small particles to higher excited states of the large particles. ${ }^{[18]}$ Recently, it was demonstrated that binary semiconducting composite materials can show strongly enhanced electronic properties with about 100 -fold higher conductance as compared to the sum of individual conductances of single-component films. ${ }^{[19]}$ Creation of highly periodic superlattices is expected not just provide the control of the homogeneity of the sample but also affect their properties. It was shown that silver nanocrystals organized into periodic cubic structures vibrated coherently ${ }^{[20]}$ and demonstrated a change in electronic transport properties. ${ }^{[21]}$

We can pursue the analogy in which nanocrystals are considered as artificial atoms, whereby nanoparticle superlattices can be viewed as a new type of solid. In inorganic crystals, many new phenomena arise in ternary and higher order compositions, where it is possible to independently tune multiple interactions. Extensive work in condensed matter physics focuses on solids composed of three and more types of atoms, giving rise, for instance, to high-temperature superconductivity, and a range of other finely tuned behaviors. The same concept should hold true

*] Dr. Elena V. Shevchenko, Dr. Dmitri V. Talapin, Dr. Shaul Aloni and Prof. A. Paul Alivisatos

The Molecular Foundry,

Lawrence Berkeley National Laboratory,

Berkeley, CA 94720 (USA).

E-mail: evshevchenko@lbl.gov

E-mail: apalivisatos@lbl.gov

Dr. Jeffrey B. Kortright,

Materials Sciences Division,

Lawrence Berkeley National Laboratory,

Berkeley, CA 94720 (USA).

[**] The work at the Molecular Foundry and Advanced Light Source was supported by the Director, Office of Science, Office of Basic Energy Science, Division of Materials Sciences and Engineering of the U.S. Department of Energy under contracts no DE-AC02-05CH1131 and DE-AC0376 SF00098. for nanocrystal-derived solids as well, where three and more component systems may exhibit a wider range of behaviours when compared to binary systems. However, a fundamental difficulty arises in the formation of ternary nanoparticle superlattices, namely it is still challenging to precisely control the sizes and especially the interactions between the NP in such a way as to promote the formation even of just binary nanoparticle supercrystals. While we can anticipate that these difficulties will be addressed over time, here we show a straightforward path to creating ternary nanoparticle superlattices while only having to control the interactions between two types of NPs.

The approach adopted here takes advantage of the recent discovery of methods to prepare one type of NP completely enclosed within a hollow shell of a second type. ${ }^{[22]}$ There are two ways in which such nested nanoparticle structures can be developed. First, a core particle such as $\mathrm{Pt}$ or $\mathrm{Au}$ can be overcoated with a second material, such as $\mathrm{Co}$ or $\mathrm{Fe}$, which are then oxidized to yield the hollow shell material. A second approach involves careful control of the oxidation of a single material, such as Fe or Co. The outer layer of NP can be oxidized under conditions where the nanoscale Kirkendall effect is used to make the shell hollow, i.e., to produce a void shell between the outer shell and the inner core. ${ }^{[22]}$ After the formation of a thin oxide layer, subsequent growth occurs by outward metal cation diffusion to the outer surface, rather than inward diffusion of oxygen anions. If the oxidation process is well controlled, it is possible to stop the oxidation before it is completed, generating a hollow shell of metal oxide or halcogenide, with a central metallic core. $^{[22-24]}$ In all cases the resulting particles are single chemical entities that are soluble and processable through ligand manipulation as with other nanocrystals, and yet they contain two distinct compositions with separate physical properties.

Nanoparticle crystallization is quite sensitive to particle size distribution, and to date there are only few multicomponent systems with appropriate particle size distribution. Here the best control over particle size distribution was achieved for iron/(iron oxide) core/(hollow shell) NPs. Thus iron/(iron oxide) core/(hollow shell) and solid Au NPs were chosen as model systems to test the possibility of growth of ternary periodic structures. Our studies on co-crystallization of these NPs revealed that quasi-ternary nanoparticle superlattices (TNSLs) isostructural with binary $\mathrm{NaCl}, \mathrm{NiAs}, \mathrm{AlB}_{2}$ and two polymorphs of $\mathrm{AB}_{13}$ : $\mathrm{NaZn}_{13}$ and cub- $\mathrm{AB}_{13}$ could be formed. ${ }^{[14,15,25]}$ Also, nanoparticle superlattices having NiAs structure were observed for the first time.

Modification of the synthetic approach developed by S. Sun, et al $^{[26]}$ led to the formation of iron -iron oxide core-hollow shell NPs with controllable size of the inner core and outer shell (Figure 1). We believe that first Fe NPs were synthesized and then, depending on the oxidation conditions, ${ }^{[23,24,26]}$ different corehollow shell or even hollow oxide structures were formed. In these particles the iron oxide shell was highly polycrystalline. Figure 1 represents a sample of $11.7 \mathrm{~nm}$ NPs taken at different oxidation states at $100{ }^{\circ} \mathrm{C}$. The temperature range $80-100{ }^{\circ} \mathrm{C}$ was found to provide fast and controllable oxidation. Increasing the temperature 
increases the oxidation rate, and at $180{ }^{\circ} \mathrm{C}$ oxidation was complete in 10 minutes. Storing of these NPs under ambient conditions does not lead to their further oxidation: no detectable changes were observed in samples aged for 4 months. As-synthesized NPs have narrow particle size distribution (std. 7-9\%) appropriate for their direct use in self-assembly experiments. Iron oxide hollow shell NPs with $\sim 4 \mathrm{~nm}$ iron core were chosen as a model component of bifunctional material in self-assembly experiments because their core-hollow shell structure is clearly visualized in TEM (Figure $1 b)$.

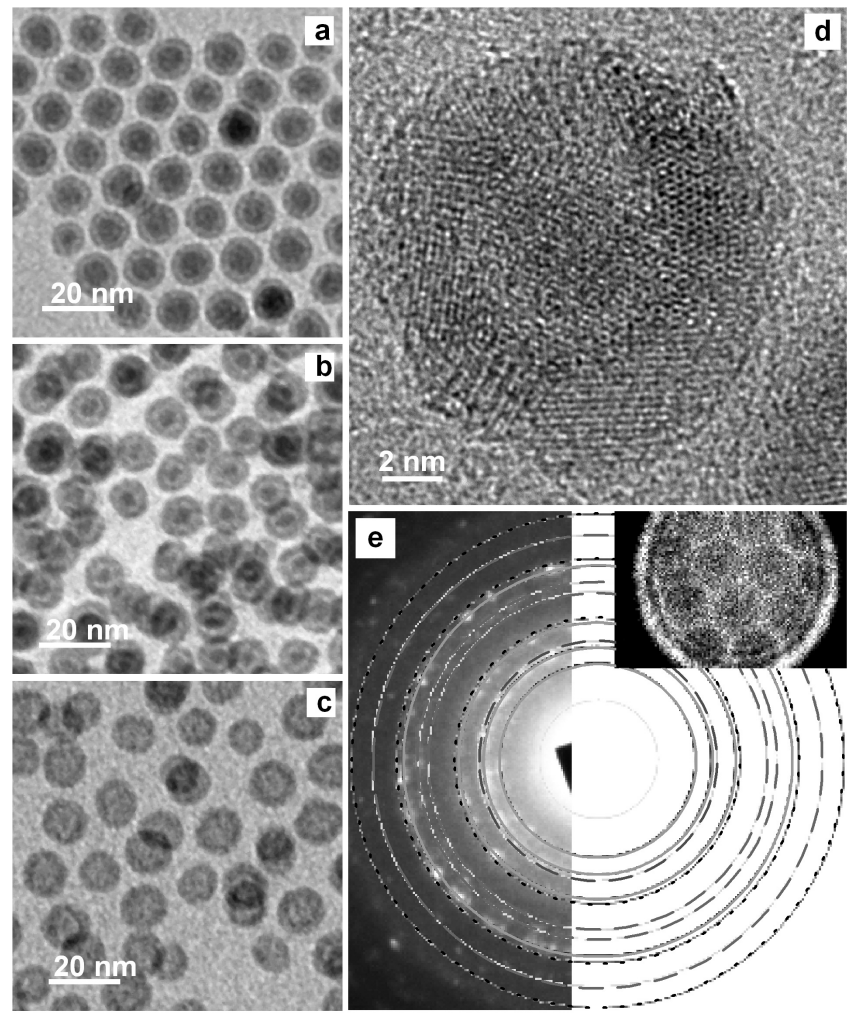

Figure 1. TEM micrographs demonstrate different stages of oxidation of Fe NPs at $100{ }^{\circ} \mathrm{C}$ : (a) $30 \mathrm{sec}$; (b) $3 \mathrm{~min}$; (c) $30 \mathrm{~min}$; (d) high resolution TEM image of hollow oxide NPs with medium ( 4 $\mathrm{nm}$ ) core; (e) selected area electron diffraction pattern: solid and dashed lines represent the strongest and weak diffraction lines of $Y$ $\mathrm{Fe}_{2} \mathrm{O}_{3}$, respectively; dots depict the position of diffraction lines of $\mathrm{Fe}$; the inset in (e) shows the area from which the nanobeam diffraction was taken.

There are several ways to identify the crystalline phase of NPs. Among the most powerful techniques are X-ray diffraction (XRD) and electron diffraction (ED). Mainly two crystalline iron oxide phases have been reported to be synthesized in non-polar solvents in $180-300{ }^{\circ} \mathrm{C}$ temperature range: $\mathrm{Fe}_{3} \mathrm{O}_{4}$ (magnetite) and $\gamma-\mathrm{Fe}_{2} \mathrm{O}_{3}$ (maghemite). ${ }^{[27-29]}$ These phases have only very slight differences in crystalline lattices. In our samples XRD revealed nearly amorphous material and ED allowed us only to exclude $\alpha-\mathrm{Fe}_{2} \mathrm{O}_{3}$ phase (hematite) (Figure 1e). The central iron core appears rather amorphous in agreement with previously reported data on solid $\mathrm{Fe} / \mathrm{Fe}_{3} \mathrm{O}_{4}$ core-shell structures. ${ }^{[26]}$ The line broadening of ED pattern resulting from the nanoscale polycrystalline grains leaves open the possibility of the co-existence of $\gamma-\mathrm{Fe}_{2} \mathrm{O}_{3}$ and $\mathrm{Fe}_{3} \mathrm{O}_{4}$ phases. Previously, it was reported that even solid highly crystalline NPs can have size-dependent mixed composition of magnetite and maghemite phases. ${ }^{[30]}$ In general, synthesis of solid and hollow iron oxide NPs has been found to be very sensitive to the oxidation conditions (temperature, oxidation agent) and capping ligands. ${ }^{[26-30]}$ This might explain the discrepancy in crystalline phase and chemical stability of iron oxide NPs synthesized by different groups. High resolution TEM investigations confirmed the polycrystalline nature of hollow coreshell and hollow shell NPs.

On average, the outer oxide shell consisted of about $7-10$ crystalline domains (Figure 1d). This means that up to $80 \%$ of atoms are located at or in the close proximity of the surface of hollow shell making the analysis of crystalline structures very complex.

Co-crystallization of two component NPs, in this case singlephase $\mathrm{Au}$ particles and core-hollow shell iron - iron oxide particles, is an elegant way to make quasi-ternary multifunctional materials, as it avoids the added complexity of crystallizing three separate types of particles. We found that self-assembly of iron/(iron oxide) core/(hollow shell) and Au NPs led to the formation of a number of quasi-ternary superlattices, isostructural with binary ionic and intermetallic compounds.

Mixing of hollow core-shell and Au NPs with 1 to 2 nanoparticle ratio followed by evaporation of toluene-TCE solvent mixture led to the formation of quasi-TNSLs isostructural with $\mathrm{NaCl}$ lattice (SG 225, Fm-3m) (Figure 2, Table 1). These structures were represented mainly by (100) and (111) projections of NaCl-type superlattices (Figures 2a, 2b). The difference in contrast in the insert to Figure $2 \mathrm{a}$ demonstrates iron/(iron oxide) core/(hollow shell) NPs with and without Au NPs at their tops. Highly defective (110) planes were also observed (Figure 2c). On average, ordering was observed across 1-5 micron. Formation of $\mathrm{NaCl}$-type superlattices was previously observed for $\mathrm{Au}-\mathrm{Ag}^{[31]}$, $\mathrm{Fe}-\mathrm{Au},{ }^{[32]} \mathrm{Fe}_{2} \mathrm{O}_{3}-\mathrm{Au}^{[14,15]}$ and $\mathrm{PbSe}-\mathrm{Pd}^{[14,15]}$ nanoparticle mixtures. Also, nanoparticle $\mathrm{AB}$ superlattices with tetragonal $\mathrm{CuAu}$ and orthorhombic $\mathrm{AB}$ superlattices were reported. ${ }^{[11,15]}$ In the case of oppositely charged polymer spheres $\mathrm{NaCl}-, \mathrm{CsCl}-$ and NiAs-type superlattices were obtained. ${ }^{[33]}$ The variety of nonclose-packed superlattices emphasizes the importance of the Columbic and dipolar interactions. Screening of these interactions opens up the possibility of the formation of other types of superlattices with the same stoichiometry. We found that cocrystallization of iron-iron oxide hollow core-shell and Au NPs with 1 to 2 nanoparticle ratio in the presence of extra dodecanethiol led to the formation of hexagonal NiAs-type superlattice (SG 194, P6 $3 / m m c$ ) (Figure 2d, Table 1). The NiAs structure can be described as ABACABAC stacking with the $\mathrm{Ni}$ atoms at the $\mathrm{A}$ sites and $\mathrm{As}$ at $\mathrm{B}$ and $\mathrm{C}$ sites. The $\mathrm{Ni}$ and As atoms have face centered cubic $(f c c)$ and hexagonally close packed $(h c p)$ environments, correspondingly. The NiAs-type was found to coexist with $\mathrm{AlB}_{2}$ type superlattice if iron/(iron oxide) core/(hollow shell) and Au NPs were mixed with particle ratio 1 to 4 and extra dodecanethiol was added (Figure 2e).

Crystallization of iron/(iron oxide) core/(hollow shell) and $\mathrm{Au}$ NPs with 1 to 4-5 particle ratio without extra capping ligands or 1 to 3 with extra oleic acid gives superlattice isostructural with $\mathrm{AlB}_{2}$ (SG 191, P6/mmm) (Figures 3a-c, Table 1). This structure has been observed for a number of different binary combinations of NPs and described in details in Refs [11,12,14]. Scanning transmission electron microscopy combined with energy dispersive $\mathrm{x}$-ray spectroscopy was applied to map the composition of the superlattice. In the high annular dark field STEM image (Figure 3d) the high contrast, bright spots are attributed the heavier element revealing the gold nanoparticle sub-superlattice. Spatial distribution of oxygen, iron and gold EDS signals further confirms the superlattice composition.

Mixing iron/(iron oxide) core/(hollow shell) and Au NPs with 1 to 6-8 particle ratio without extra capping ligands or with extra oleyl amine gives superlattice isostructural with $\mathrm{NaZn}_{13}{ }^{[14,15]}$ and 
its polymorphous cuboctahedral form cub- $\mathrm{AB}_{13}{ }^{[25]}$ (Figures 2f,g, Table 1). As in the case of solid binary NPs these structures always appear co-existing with each other with a preferred orientation relationship: usually (001) projection corresponds to cub- $\mathrm{AB}_{13^{-}}$and (110) projection is the most characteristic for $\mathrm{NaZn}_{13}$-type superlattices.

Entropy is considered as the sole driving force for selfassembly of non-interacting hard spheres favouring most densely packed structures. ${ }^{[34]}$ However, we came across understanding that NPs are much more complex objects compared to hard noninteracting spheres and that many interactions (Columbic, van der Waals, dipolar, etc) can occur between particles in solution and between particles and the substrate. Recently it was shown that the electrostatic interactions between oppositely charged particles can play important role in determining the type of crystalline lattice. ${ }^{[13,14,25]}$ In the case of NPs, Coulombic interactions compete with dipolar and van der Waals interactions. ${ }^{[14,15]}$ Roughly equal contributions of these interactions lead to the diversity in binary superlattices, ${ }^{[25]}$ while their complexity continues to challenge our theoretical understanding of nanoscale crystallization.

Diverse interactions at the nanoscale make possible stabilization of non-close packed structures. For instance, even in the case of monodisperse NP, we found that in addition to well known cubic (ABCABC...layer stacking) and hexagonal ( $\mathrm{ABABAB} \ldots$ layer stacking) close-packing, simple hexagonal arrangement of particles with AAA... stacked hexagonally stacked layers of NPs is possible due to strong dipole-dipole coupling between the NPs. ${ }^{[35]}$ In binary mixtures the nature of nanoparticle interactions is even more complex and crystallization is guided by a combination of competing Coulombic, dipolar, and van der Waals forces. ${ }^{[15]}$ These interactions allow superlattice formation to be dependent on a number of tuneable parameters such as temperature, charge states, etc. Combining the same iron/(iron oxide) core/(hollow shell) and Au NPs and adjusting the experimental conditions we were able to make superlattices with packing density ranged from 0.5 up to 0.76 (Table 1). Such significant difference in packing density rules out the validity of the hard sphere approximation in the formation of NP superlattice and confirms the importance of interparticle interactions. The crystalline nature of NPs gives us an access to a library of NPs with different shapes. Also different complex geometries of multifunctional NPs such as core-shell, core-hollow shells, dumbbells, etc can be obtained. Proper adjustment of composition of multicomponent NPs might make possible effective control over dipole-dipole, charge-dipole and, especially, van der Waals interactions. Also this can provide additional degrees of freedom not available in the world of atoms, ions and polymer materials. The difficulties encountered in co-crystallizing more than two types of NPs can be overcome by using multicomponent nanoparticles as the superlattice building blocks. The formation of ternary nanoparticle superlattices greatly extends the range and combinations of possible material types which can be intermixed on the nanoscale. The nested topology of the components may influence a range of properties. We believe self-assembly of complex "artificial atoms" (nanoparticle building blocks) may provide an efficient route to the formation of materials and metamaterials not observed in nature. Self-assembly of bi- functional and uni-functional NPs seems to be the simplest model system to study the contribution of different particle-particle interactions as a function of their composition. Our work suggests that further progress in synthesis of bi- and multi-functional NPs will facilitate the access to novel families of three and more component nanoparticle superlattices.

Table 1. Lattice density of different structures for nanoparticle size ratio 0.44 .

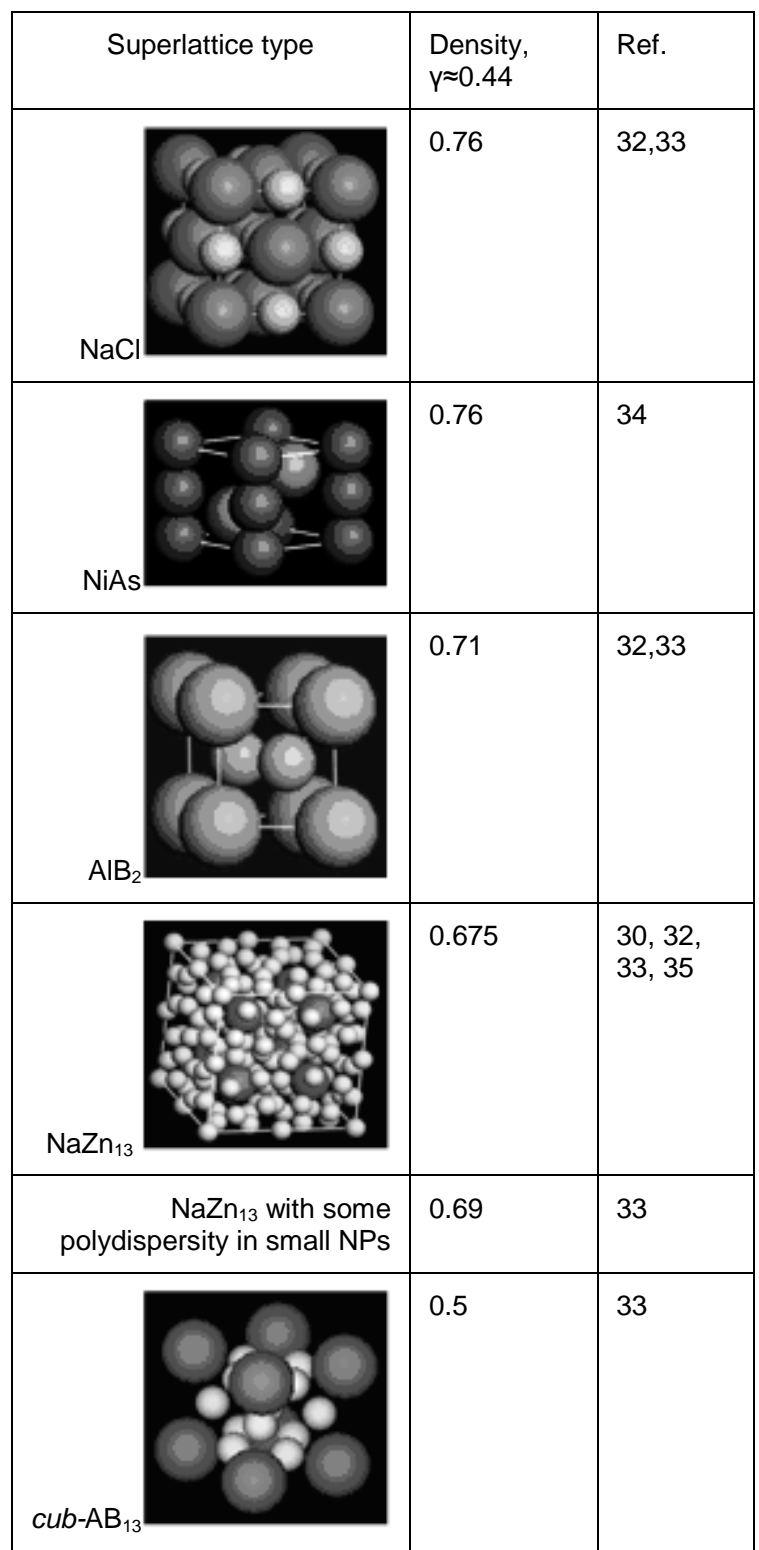

$11.7 \mathrm{~nm} \mathrm{Fe} / \mathrm{Fe}_{3} \mathrm{O}_{4}$ and $4.5 \mathrm{~nm}$ Au NPs stabilized by oleyl amine and dodecanethiol respectively (the distance between nanoparticles in $2 \mathrm{D}$ array of $\mathrm{Fe} / \mathrm{Fe} 3 \mathrm{O} 4$ is $1.8 \mathrm{~nm}$ while monodisperse Au NPs are $1.5 \mathrm{~nm}$ apart). 

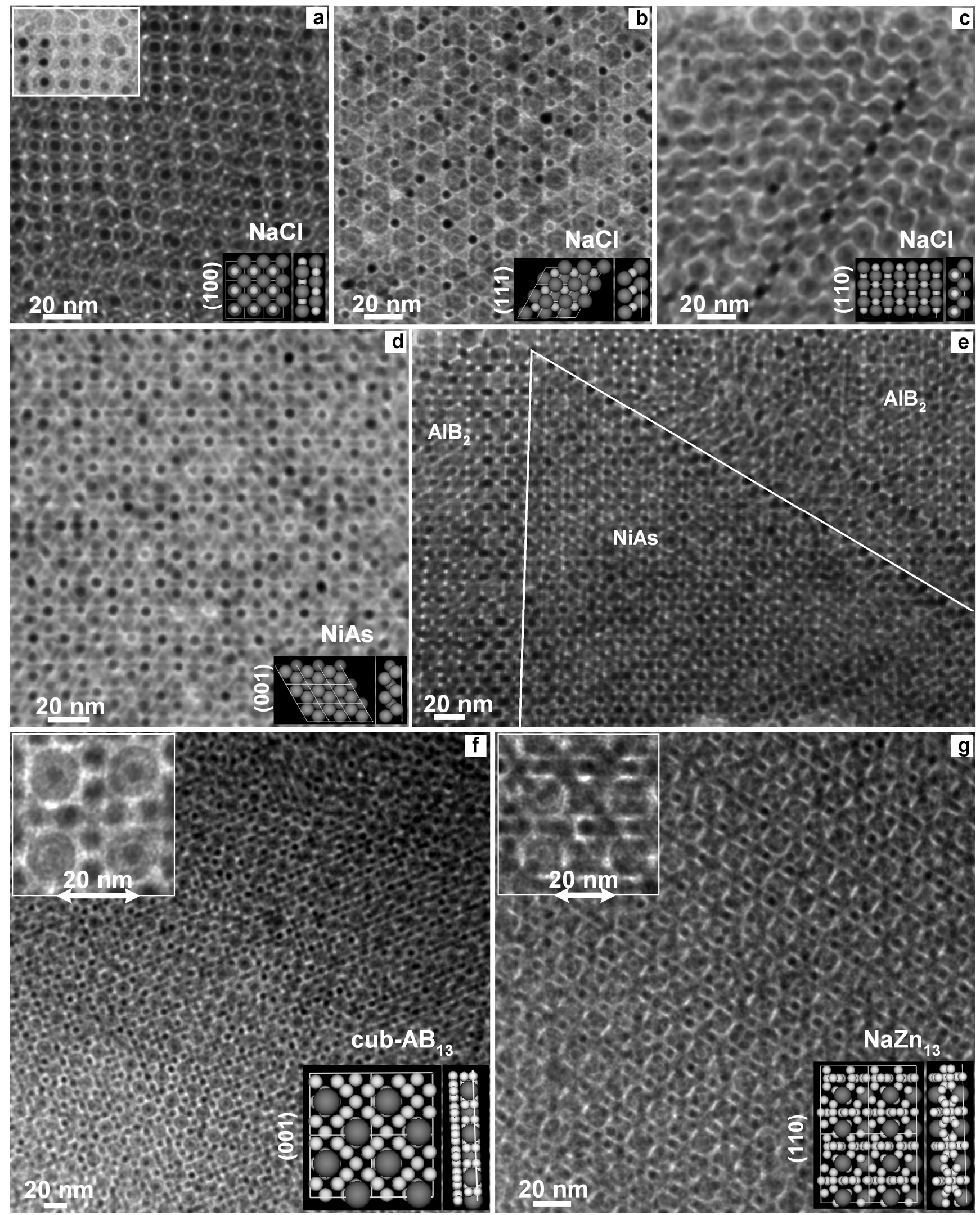

Figure 2. TEM micrographs of different quasi-TNSLs formed by $11.7 \mathrm{~nm}$ iron/(iron oxide) core/(hollow shell) NPs and $4.5 \mathrm{~nm}$ Au NPs and isostructural with (a-c) NaCl ((a), (b), (c) represent (100), (111) and (110) projections respectively); (d) (001) projection of quasi TNSLs isostructural with NiAs; (e) NiAs-type quasi TNSLs co-exist with AlB $_{2}$-type phase; $(f)(001)$ projection of cub-AB ${ }_{13}$ and $(g)(110)$ projection of $\mathrm{NaZn}_{13}$-type superlattices. Low right inserts into TEM images represent graphical sketches of corresponding projections and minimum numbers of layers in such planes, leading to the formation of the patterns identical to the observed ones. 

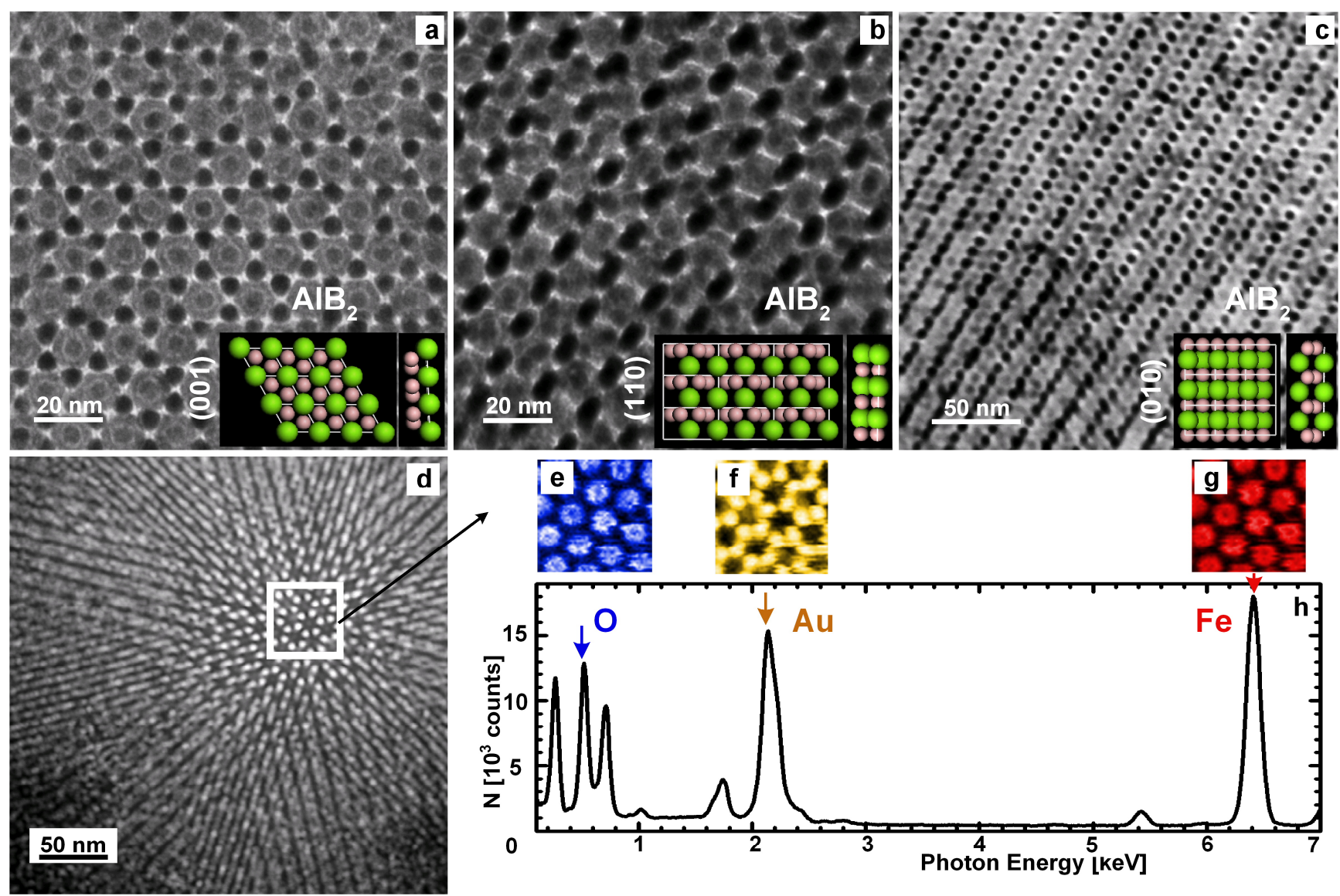

Figure 3. TEM micrographs of (a) (001), (b) (110) and (c) (010) projections of quasi TNSLs isostructural with $\mathrm{AlB}_{2}$; (d) high angle annular dark field STEM image of $\mathrm{AlB}_{2}$-type superlattice. Elemental maps of $(\mathbf{e})$ oxygen, (f) gold and $(\mathbf{g})$ iron obtained by recording spatial distribution of respective EDS signal intensities; (h) averaged EDS spectrum taken form $\mathrm{AlB}_{2}$-type assembly

\section{Experimental}

Chemicals: Octadecene, toluene, ethanol, tetrachloroethylene (TCE), oleic acid, oleylamine $\mathrm{AuCl}_{3}, \mathrm{Fe}(\mathrm{CO})_{5}$, sodium borohydrate, dodecyldimethylammonium bromide (DDAB), were purchased from Aldrich. Oleic acid and oleylamine were of technical degree of purity. All chemicals were used as received.

Nanoparticle Synthesis: iron/(iron oxide) core/(hollow shell) and Au NPs were synthesized in nonpolar solvents and stabilized with amphiphilic molecules containing long $\mathrm{C}_{17}$ and $\mathrm{C}_{12}$ alkyl chains, respectively. Hollow Iron-Iron Oxide core-shell nanoparticles: We used modified method of S. Sun developed for synthesis of Fe NPs with densely attached $\mathrm{Fe}_{3} \mathrm{O}_{4}$ shell. ${ }^{[26]}$ Briefly, $0.3 \mathrm{ml}$ of oleylamine was dissolved in octadecene $(20$ $\mathrm{mL}$ ). The solution was kept under vacuum at $120{ }^{\circ} \mathrm{C}$ for $30 \mathrm{~min}$ and than the temperature was raised to $180{ }^{\circ} \mathrm{C}$. At this temperature, $0.7 \mathrm{~mL}$ of $\mathrm{Fe}(\mathrm{CO})_{5}$ were injected into the solution and kept at $180{ }^{\circ} \mathrm{C}$ for $30 \mathrm{~min}$. After that the colloidal solution was cooled to $100{ }^{\circ} \mathrm{C}$ and the air was passed through the solution. Depending on the oxidation time, different hollow core-shell iron-iron oxide or hollow iron oxide structures were formed. After oxidation the NPs were precipitated by ethanol followed by centrifugation and washed out from the excess of oleylamine using toluene - ethanol solvent-nonsolvent combination. Au nanoparticles: The detailed synthesis of Au NPs is described in Refs [15,40].

Preparation of Nanoparticle Superlattices: To aid structural analysis, the quasi ternary superlattices were grown on electron transparent substrates such as amorphous carbon film. Ultrathin carbon supported by 300 mesh copper grids (type A, Ted Pella, Inc.) was cleaned from formvar by dipping for 15 seconds in toluene. Substrates (TEM grids) were placed in a glass vial with colloidal solution of NPs. Mixtures of toluene with TCE were used as solvents (1:1 by volume). Colloidal solutions of Au and iron-iron oxide in toluene were mixed with TCE as diluting solvent $(\sim 1: 1$ by volume). This solvent mixture provided the best reproducibility and area of ordering. In a typical experiment $\sim 12-20 \mu \mathrm{L}$ of solution were evaporated per grid. The vial was tilted by $\sim 60^{\circ}$. The deposition of periodic structures occurred on carbon side upon evaporation of the solvent at $40-50{ }^{\circ} \mathrm{C}$ under reduced pressure $(\sim 2.5 \mathrm{kPa})$. The increased temperature is essential because of tendency of Au NPs to aggregation at room temperature. To direct the self-assembly, $2 \mu \mathrm{L}$ of TCE solution of oleic acid or dodecanethiol ( $1 \mu \mathrm{L}$ of capping ligands in $100 \mathrm{~mL}$ of TCE) were added to the colloidal solution of NPs.

Characterization of nanoparticles and their superlattices: A Tecnai G2 S-Twin electron microscope (TEM) operating at $200 \mathrm{kV}$ was used for imaging $\mathrm{Au}$ and iron/(iron oxide) core/(hollow shell) NPs and their superstructures. High resolution TEM and high angle angular dark field scanning transmission microscopy (HAADF STEM) studies and mapping of the elements were performed using JEOL 2100-F $200 \mathrm{kV}$ FieldEmission Analytical Transmission Electron Microscope equipped with an Oxford energy dispersive X-ray spectrometer (EDS) and Gatan imaging filter for energy loss spectroscopy (EELS). Three-dimensional description of the superlattices is based on surveying large regions of the samples and recording a series of two dimensional (2D) projections down the major symmetry axes. To assign the observed structures to crystallographic space groups, we built three dimensional (3D) models of different lattices using Accelrys MS Modeling 3.1 software and cleaved them along different crystallographic directions. The TEM images were compared with simulated projections to match the symmetry of our superlattices 
Received: ((will be filled in by the editorial staff))

Revised: ((will be filled in by the editorial staff))

Published online: ((will be filled in by the editorial staff $)$ )

[1] H. W. Gu,; R. K. Zheng, X. X.Zhang, B. Xu J. Am. Chem. Soc. 2004 126, 5664.

[2] T. Pellegrino, A. Fiore, E. Carlino, C. Giannini, P. D. Cozzoli, G. Ciccarella, M. Respaud, L. Palmirotta, R. Cingolani, L. Manna, J. Am. Chem. Soc. 2006, 128, 6690 .

[3] H. Yu, M.; Chen, P. M. Rice, S. X. Wang, R. L. White, S. Sun. Nano Lett. 2005, 5, 379.

[4] H. W. Gu, Z. M.Yang, J. H. Gao, C. K. Chang, B.Xu. J. Am. Chem. Soc. 2005, 127, 34 .

[5] W. Shi, H. Zeng, Y. Sahoo, T. Y. Ohulchanskyy, Y. Ding, Z. L. Wang, P. N. Prasad, Nano Lett. 2006, 6, 875.

[6] D. J. Milliron, S. M. Hughes, Y. Cui, L. Manna, J. B. Li, L. W. Wang, A. P.Alivisatos. Nature 2004, 430, 190.

[7] C. Pacholski, A. Kornowski, H. Weller. Angew. Chem., Int. Ed. 2004, 43, 4774.

[8] J. Yang, H. I. Elim, Q. Zhang, J. Y.Lee, W. Ji. J. Am. Chem. Soc. 2006, 128,11921

[9] M. Casavola, V. Grillo, E. Carlino, C. Giannini, F. Gozzo, E. Fernandez Pinel, M. A. Garcia, L. Manna, R. Cingolani, P. D. Cozzoli. Nano Lett. 2007, 7, 1386-1395.

[10] R. Buonsanti, V. Grillo, E. Carlino, C. Giannini, M.L. Curri, C. Innocenti, C. Sangregorio, K. Achterhold, F.G. Parak, A. Agostiano, P.D. Cozzoli. J. Am. Chem. Soc. 2006, 128, 16953.

[11] C.J. Kiely, J. Fink, M.Brust, D. Bethel, D.J. Schiffrin. Nature 1998, 396, 444.

[12] F.X. Redl, K.-S. Cho, C.B. Murray, S..O'Brien. Nature 2003, 423, 968.

[13] M. Kalsin, M.; Fialkowski, M. Paszewski, S. K. Smoukov, K. J. M. Bishop, B. A. Grzybowski, Science 2006, 312, 420.

[14] E. V. Shevchenko, D. V. Talapin, S. O'Brien C. B. Murray. J. Am. Chem Soc. 2005, 127, 8741.

[15] E. V. Shevchenko, D. V. Talapin, N. A. Kotov, S. O’Brien, C. B. Murray. Nature 2006, 439, 55.

[16] H. Zeng, J. Li, J. P. Liu, Z. Wang, S. Sun Nature 2002, 420, 395.

[17] R. Skomski, J. M. D.Coey. Phys. Rev. B 1993, 48, 15812.

[18] C.R.Kagan, C.B.Murray, M.G. Bawendi. Phys.Rev B 1996, 54, 8633.
[19] J.J. Urban, D. V. Talapin, E. V. Shevchenko, C. R. Kagan, C. B. Murray. Nature Materials 2007, 6, 115.

[20] A. Courty, A. Mermer, P.A. Albouy, E. Duval, M.P.Pileni , Nat. Mater, 2005, 4, 395

[21] A. Taleb, F. Silly, A.O. Gusev, F. Charra, M.P. Pileni, Adv. Mater. 2000, $12,633$.

[22] Y. Yin, R. M. Rioux, C. K. Erdonmez, S. Hughes, G. A. Somorjai, A. P. Alivisatos Science 2004, 304, 711.

[23] S. Peng, S. Sun. Angew. Chemie Int. Ed. 2007, 46, 4155.

[24] A. Cabot, V. F. Puntes, E. Shevchenko, Y. Yin, L. Balcells, M. A. Marcus, S. M. Hughes, A. P. Alivisatos. J. Am. Chem. Soc. 2007, 129, 10358.

[25] E.V. Shevchenko, D.V. Talapin, C. B. Murray, S. O'Brien. J. Am. Chem. Soc. 2006, 128, 3620.

[26] S. Peng, C. Wang, J. Xie, S. Sun. J. Am. Chem. Soc. 2007, 128, 10676.

[27] T. Hyeon, S. S. Lee, J. Park, Y. Chung, H. B. Na. J. Am. Chem. Soc. 2001, 123,12798

[28] K. Woo, J.Hong, S. Choi, H.-W. Lee, J.-P. Ahn, C. S. Kim, S. W. Lee. Chem. Mater., 2004, 16, 2814.

[29] S. Sun, H. Zeng, D. B. Robinson, S. Raoux, P. M. Rice, S. X. Wang, G. Li. J. Am. Chem. Soc., 2004, 126, 273.

[30] J, Park, K. An, Y. Hwang, J.-G. Park, H.-J. Noh, J.-Y. Kim, J.-H. Park, N.-M.;Hwang, T. Hyeon, Nature Mater., 2004, 3, 891.

[31] C. J. Kiely, J. Fink, J. G. Zheng, M.. Brust, D. Bethell, D. J. Schiffrin, Adv. Mater. 2000, 12, 640.

[32] A.E. Saunders, B.A. Korgel. ChemPhysChem. 2005, 6, 61.

[33] M.E. Leunissen, C.G. Christova, A.-P. Hynninen, C.P. Royall A.P. Campbell, A. Imhof, M. Dijkstra, R. van Roij, A. van Blaaderen. Nature 2005, 437, 235.

[34] M.D. Eldridge, P.A. Madden, D. Frenkel. Nature 1993, 365, 35.

[35] D.V. Talapin, E.V. Shevchenko, C. B. Murray, A.V. Titov, P. Kral. Nano Lett. 2007, 7, 1213.

[36] X. Cottin, P.A. Monson. J. Chem. Phys. 1995, 102, 3354.

[37] M.J. Murray, J.V. Sanders. Phyl. Mag. A 1980, 42, 721.

[38] N. Hunt, R. Jardine, P. Bartlett. Phys. Rev. E 2000, 62, 900.

[39] M.D. Eldridge, P.A. Madden, D. Frenkel. Mol. Phys. 1993, 79, 105.

[40] B.L.V. Prasad, S. I. Stoeva, C.M. Sorensen, K.J. Klabunde. Langmuir 2002, 18, 7515 . 
Table of Contents

\section{COMMUNICATION}

Three component nanoparticle superlattices isostructural with binary ionic and intermetallic compounds are obtained by co-crystallization of multicomponent nanoparticles. Selfassembly of multicomponent nanoparticles greatly extends the combinations of possible materials types which can be intermixed on the nanoscale.

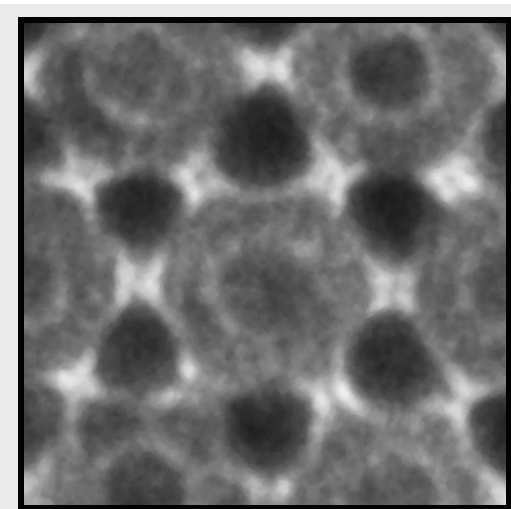

Quasi-Ternary nanoparticle superlattices

Elena V. Shevchenko, Jeffrey Kortright, Dmitri V. Talapin, Shaul Aloni and A.

Paul Alivisatos.

Page No1. - Page No6.

Quasi-ternary nanoparticle superlattices through nanoparticle design. 